\title{
Sistematización de la evaluación de riesgo de violencia con instrumentos de juicio profesional estructurado en Cuenca, Ecuador
}

\author{
Juana V. Ochoa Balarezo ${ }^{1}$, Ximena K. Guillén ${ }^{1}$, Dione Ullauri Ortega ${ }^{2}$, Juana Narváez ${ }^{2}$, Elizabeth \\ León-Mayer ${ }^{3}$, Jorge O. Folino ${ }^{4}$ \\ ${ }^{1}$ Psicóloga, Instituto de Criminología, Criminalística e Intervención Psicosocial Familiar de la \\ Universidad de Cuenca, Ecuador. \\ 2 Trabajadora Social, Instituto de Criminología, Criminalística e Intervención Psicosocial Familiar de \\ la Universidad de Cuenca, Ecuador. \\ ${ }^{3}$ Doctora en Ciencias de la Salud, Facultad de Ciencias Médicas de la Universidad Nacional de La \\ Plata, Argentina. \\ ${ }^{4}$ Doctor en Medicina y Profesor de Psiquiatría, Facultad de Ciencias Médicas de la Universidad \\ Nacional de La Plata, Argentina; Investigador del Proyecto Prometeo, SENESCYT, en la \\ Universidad de Cuenca, Ecuador.
}

Autores para correspondencia: juana.ochoab@ucuenca.edu.ec, jorgefolino@fibertel.com.ar

Fecha de recepción: 9 de noviembre 2016 - Fecha de aceptación: 16 de marzo 2017

\section{RESUMEN}

Introducción: La manera en que los profesionales de la salud mental realizan la evaluación del riesgo de violencia y la planificación de intervención tiene impacto en las decisiones judiciales, en el bienestar social y en la responsabilidad profesional.

Objetivos: Determinar la confiabilidad de instrumentos de evaluación de psicopatía e instrumentos de juicio profesional estructurado para la evaluación de riesgo de violencia en el ámbito del Instituto de Criminología, Criminalística e Intervención Psicosocial Familiar de la Universidad de Cuenca, Ecuador.

Método: Duplas de psicólogos y de trabajadores sociales, previamente entrenados, evaluaron simultáneamente a 37 casos derivados al instituto con instrumentos de evaluación de riesgo de violencia general-HCR-20- y conyugal -SARA ${ }^{1}$ - y de evaluación de psicopatía -Hare PCL-R ${ }^{2}$-. Se calcularon indicadores de concordancia y de consistencia interna.

Resultados: La concordancia de la valoración del riesgo de violencia hacia la pareja fue excelente -CCI: 0.76 y 0.90 para las psicólogas y las trabajadoras sociales respectivamente-. Los indicadores para las diferentes secciones del HCR-20 y del SARA también fueron excelentes, con rango entre 0.75 y 0.94 . El indicador para el PCL-R total fue 0.96 .

Conclusiones: Los resultados permiten sostener que el uso de estos instrumentos en el ámbito ecuatoriano, con el correspondiente entrenamiento, resulta confiable. El uso de estos instrumentos contribuye a la sistematización y transparencia del procedimiento y al resguardo de la responsabilidad profesional.

Palabras clave: Riesgo de violencia, evaluación, psicopatía, violencia conyugal.

\section{ABSTRACT}

Introduction: The way professionals of mental health carry out violence risk assessment and intervention planning has an impact on judicial decisions, social wellbeing and professional responsibility.

\footnotetext{
1 SARA: Spousal Assault Risk Assessment

2 Hare PCL-R: Hare Psychopathy Checklist-Revised
} 
Objectives: To determine the reliability of the psychopathy evaluation instruments and structured professional guides in violence risk assessment used at the Institute of Criminology and Family Psychosocial Intervention of the University of Cuenca, Ecuador.

Method: Previously trained pairs of psychologists and social workers assessed simultaneously 37 cases, who were transferred to the Institute, using structured violence risk assessment instruments -HCR 20 and SARA- and psychopathy evaluation instrument -Hare PCL-R-. Indicators of internal agreement and consistency were calculated.

Results: The agreement of the assessment of the risk of violence towards the couple was excellent. The intraclass coefficient was respectively 0.76 and 0.90 for psychologists and social workers. The indicators for the different sections of the HCR-20 and SARA were also excellent, ranging between 0.75 and 0.94 . The indicator for PCL-R total was 0.96 .

Conclusions: The results support the reliability of these instruments in Ecuador provided the users receive adequate training. The use of these instruments contributes to the systematization and transparency of the risk assessment procedures and the protection of the professional responsibility.

Keywords: Violence risk, assessment, psychopathy, spouse violence.

\section{INTRODUCCIÓN}

La prevención de la violencia es una de las prioridades para la agenda de desarrollo sustentable de la Organización Mundial de la Salud y un compromiso de sus estados miembros (World Health Organization, 2014). Como en otras partes del mundo, en Ecuador se vienen realizando aportes legislativos tendientes a contribuir con la prevención de la violencia, tal como se verifica en el capítulo tercero de la Constitución de la República (Asamblea Constituyente, 2008), en el Código de la Niñez y Adolescencia (Congreso Nacional, 2003) y en el Código Orgánico Integral Penal (Asamblea Nacional República del Ecuador, 2014). Ese marco legislativo orienta la aplicación de diversas acciones concretas que son gestionadas por operadores de la justicia y funcionarios de instituciones conexas.

Las acciones judiciales, más allá de su efecto restableciendo derechos, impactan directamente en la probabilidad de futura violencia, es decir, tiene un efecto sobre la prevención. Así ocurre cuando un juez de familia dispone una medida de exclusión familiar en algún caso de violencia conyugal, cuando un juez penal dispone de una medida de seguridad para algún sobreseído por inimputabilidad, o cuando un juez de la niñez y adolescencia dispone la inserción de alguien violento en un programa de atención especializada. Ese proceso de gestión está mediado por la consideración de las infinitas alternativas que la realidad social presenta y que no están especificadas en las normas, por lo que el proceso de decisión judicial requiere de la apreciación de lo justo, lo apropiado, lo posible y lo deseable entre otros condicionantes. Para tan delicadas y complejas decisiones, los operadores de la justicia suelen informarse a través de expertos que realizan tareas técnicas pertinentes. Cuando las decisiones se relacionan con la violencia, son pertinentes tareas de evaluación de los factores de salud mental y de las condiciones psicosociales que pueden influir en la causalidad y prevención de la violencia y en el tratamiento de sus consecuencias.

Este artículo trata sobre el modo en que se realiza esa tarea técnica. Específicamente, con la meta de contribuir con la optimización de la evaluación de riesgo de violencia en el Ecuador, se planteó una investigación con el objetivo de poner a prueba la confiabilidad de instrumentos de evaluación de psicopatía e instrumentos de juicio profesional estructurado para la evaluación de riesgo de violencia en el ámbito del Instituto de Criminología, Criminalística e Intervención Psicosocial Familiar de la Universidad de Cuenca, Ecuador. En este artículo se informan las evidencias obtenidas y se discuten sus implicancias en la práctica profesional.

\section{El desarrollo de la evaluación de riesgo de violencia}

La tradicional aproximación a la evaluación de riesgo de violencia, que fue predominante durante los siglos XIX y XX, estuvo modelada en el mundo por la evaluación clínica no estructurada que, en su 
mejor versión, intentó hacer confluir perspectivas fenomenológicas, existenciales y criminológicas que permitieren representarse la conducta futura y los riesgos implícitos en la misma. Ese modelo, si bien podría tener fortalezas en manos del clínico perspicaz y cuidadoso, fue tempranamente blanco de críticas en base al déficit en sistematización, al riesgo de evaluaciones y predicciones idiosincrásicas y a la carencia de contexto temporal (Ennis \& Litwack, 1974; Monahan, 1981).

La superación de aquellos déficits se buscó de diversas maneras. En cuanto al marco temporal, en la década de los 80 se propuso focalizar la predicción de violencia en el corto plazo (Tardiff, 1989) y posteriormente se concretaron estudios longitudinales que ofrecieron precisiones a diferentes plazos de seguimiento (Quinsey et al., 1999). También se reconoció la importancia de utilizar múltiples fuentes de información para obtener más precisión en las evaluaciones e investigaciones (Mulvey et al., 1994) y se aplicaron técnicas estadísticas reductoras del efecto problemático que tienen las bajas tasas basales de violencia en la precisión predictiva (Harris et al., 1993; Mossman, 1994; Steadman et al., 1998).

Diversos estudios obtuvieron evidencias sobre la relación de la violencia con diversos factores de salud mental, como por ejemplo, el abuso de sustancias (Swanson et al., 1994), los trastornos psicóticos (Link et al., 1994) y la psicopatía (Hare \& Hart, 1993; Harris et al., 1991; Quinsey et al., 1995; Rice \& Harris, 1992; Webster et al., 1997b).

Finalmente, se formularon múltiples instrumentos para la evaluación de riesgo (de Vogel et al., 2007; Douglas et al., 1996; Hanson, 1997; Kropp \& Hart, 2000; Monahan et al., 2000; Quinsey et al., 1999; Webster et al., 1997a) que permitieron, no sólo mejorar la sistematización sino también, comparar sus eficacias predictivas (Singh et al., 2011).

Internacionalmente, los enfoques a la evaluación del riesgo de violencia de las últimas décadas pueden esquematizarse en unos pocos modelos, tales como el actuarial, el anamnéstico, el del juicio profesional estructurado -JPE- y el compuesto (Douglas \& Kropp, 2002; Falzer, 2013; Folino, 2004b; Folino \& Escobar-Córdoba, 2004). Mientras desde el primer modelo se intenta estimar el riesgo de violencia en términos relativos, es decir, comparando al sujeto con un grupo de referencia o normatizado, desde el segundo se focaliza en los aspectos idiográficos y en las circunstancias únicas del sujeto evaluado. El modelo JPE propone realizar juicios lo más específicos posibles, incluyendo dimensiones tales como la naturaleza, la severidad, la inminencia, la verosimilitud y la frecuencia de la violencia basándose en alguna guía que oriente o estructure el proceso de obtención de información y de conclusión ( Douglas \& Ogloff, 2003). El modelo compuesto, por su parte, está orientado a integrar los modelos actuarial, anamnéstico y JPE, y la disponibilidad de dispositivos concretos de intervención (Folino, 2004b). Entre los instrumentos sustentados en el JPE que obtuvieron amplia difusión y aceptación internacional se destacan el HCR-20 (Webster et al., 1995; Webster et al., 1997b) y el SARA (Kropp et al., 1995).

La bibliografía internacional reciente pone de manifiesto la amplia preocupación de los autores por la sistematización de las tareas de evaluar el riesgo de violencia y de planificar y monitorear la intervención destinada a prevenir su reiteración. Es destacable que en esa literatura predominen los artículos sobre instrumentos que guían la evaluación y sobre la puesta a prueba de la respectiva confiabilidad, validez y utilidad práctica (Goldstein et al., 2015). La preocupación de la comunidad científica incidió también en la revisión de varios de los instrumentos (Harris et al., 2015; Rice et al., 2013; Douglas et al., 2013) y más recientemente aún en la traducción y adaptación al español de algunos de ellos (Douglas et al., 2015).

En consonancia con los avances de otras latitudes, en algunas regiones de América Latina vienen desarrollándose investigaciones sobre el tema. En Argentina, un programa de investigación con sede central en La Plata y vínculos entre la Procuración General de la Provincia de Buenos Aires, la Universidad Nacional de La Plata y otros centros forenses internacionales, ha producido diversos estudios describiendo la población penitenciaria en términos de factores de riesgo y la tasa de recidiva basal; poniendo a prueba la confiabilidad y validez predictiva de diversos instrumentos y evaluando la relación entre recidiva violenta con factores dinámicos y con indicadores bioquímicos de abuso de sustancia ( Folino et al., 2002; Folino \& Marchiano, 2002; Folino et al., 2003; Folino et al., 2004a; Folino et al., 2004b; Folino, 2005 \& 2015; Folino \& Castillo, 2006; Folino et al., 2007a; Gutierrez et al., 2012). En Brasil, especialmente en Porto Alegre, se produjeron estudios descriptivos de población forense y también estudios intramuros de confiabilidad y de validez predictiva de instrumentos 
específicos (de Borba Telles et al., 2009a; de Borba Telles et al., 2009b; de Borba Telles et al., 2010; de Borba Telles et al., 2012; Teitelbaum, 2010). En Chile se realizaron diversas contribuciones al conocimiento local de los factores de riesgo de violencia, especialmente la psicopatía y también en jóvenes infractores (Alarcón et al., 2012; León Mayer \& Folino, 2009; León-Mayer et al., 2010; LeónMayer et al., 2013; León-Mayer et al., 2014a; León-Mayer et al., 2014b; León-Mayer et al., 2015; Pérez-Luco et al., 2012; Vasconcellos \& León-Mayer, 2006; Zúñiga \& Mayer, 2008). También se cuenta con aportes colombianos a la temática, especialmente en lo que se refiere a violencia juvenil (Acero González et al., 2007; Escobar-Córdoba et al., 2015). En Ecuador no hay antecedentes de práctica profesional sistemática con instrumentos de evaluación de riesgo de violencia y solamente se cuenta con el aporte descriptivo de población penitenciaria realizado por Ochoa-Rosales (OchoaRosales, 2007). La relevancia de la temática en este país y la necesidad de sistematización fue destacada en recientes estudios (Ochoa et al., 2016; Ullauri et al., 2016).

Mayor información sobre el desarrollo de la temática en América Latina puede encontrarse en otras publicaciones (Folino et al., 2016; Goldstein et al., 2015; Singh et al., 2013), pero con esta breve descripción se hace muy evidente la necesidad de avanzar en la investigación específica en Ecuador y poner a disposición de los profesionales locales información que les permita optimizar y respaldar sus prácticas con evidencias empíricas.

\section{El contexto del estudio}

El estudio se realizó en el Instituto de Criminología, Criminalística e Intervención Psicosocial Familiar de la Facultad de Jurisprudencia y Ciencias Políticas y Sociales de la Universidad de Cuenca, Ecuador, en el marco de un proyecto de investigación patrocinado por el Programa Prometeo de la Secretaría de Educación Superior, Ciencia, Tecnología e Innovación de la República del Ecuador. La creación del instituto data de 1984 (Consejo Universitario de la Universidad de Cuenca, 1984) y el establecimiento de sus actuales funciones académicas, de cooperación con instituciones judiciales, sanitarias, penitenciarias y educativas y de intervención psicosocial familiar se hizo más recientemente (Consejo Universitario de la Universidad de Cuenca, 2011) en consonancia con a las nuevas exigencias del reordenamiento jurídico constitucional del país (Asamblea Constituyente, 2008; Asamblea Nacional República del Ecuador, 2014; Congreso Nacional, 2003). El instituto responde a requerimientos de servicios periciales y de intervención provenientes de instituciones judiciales, municipales y, también, directamente de personas de la comunidad. La problemática tratada está fuertemente atravesada por la violencia y diversos riesgos para niños, adolescentes y mujeres. El perfil del servicio es socialmente valioso pero muy exigente profesionalmente, pues demanda capacitación profesional en temas de violencia y muchos cuidados de la responsabilidad y ética profesionales (Ullauri et al., 2016).

\section{MATERIAL Y MÉTODOS}

Diseño y procedimiento: El estudio se diseñó para examinar la concordancia entre evaluadores al utilizar instrumentos de juicio profesional estructurado para la evaluación de riesgo de violencia. Dos psicólogas y dos licenciadas en trabajo social revisaron simultáneamente con aproximación de equipo 37 personas ingresadas consecutivamente al Instituto durante el período Abril 2015 a Marzo de 2016. Inicialmente se realizó el registro de puntuación en base a los instrumentos independientemente y luego el registro de puntuación consensuada. A los efectos de la puntuación se distribuyeron los ítems de acuerdo a la mayor pertinencia profesional. Los profesionales, que ya contaban con amplia experiencia en entrevistas de evaluación fueron, además, entrenados en el uso de los instrumentos. La carga de datos se realizó con chequeo cruzado.

Instrumentos: Se utilizaron el HCR-20 ( Folino, 2003; Webster et al., 1997); el SARA (Folino, 2004a; Kropp et al., 1995) y el Hare PCL-R (Hare, 2003a; León-Mayer, 2009). El procedimiento para obtener la información necesaria para su uso es una estrategia de métodos múltiples, reflejando las exigencias que plantea la pluridimensión del fenómeno violento y los sesgos informativos que el experto suele enfrentar en la tarea de evaluación. 
La HCR-20 fue originariamente publicada en Canadá (Webster et al., 1995; Webster et al., 1997). La versión utilizada fue la traducida y adaptada en Argentina (Folino, 2003). No es un test psicológico formal, sino una guía para la evaluación del riesgo de violencia, especialmente en ámbitos en que haya un número muy alto de personas con historias de violencia y presunción de enfermedad mental o trastorno de personalidad. Por lo tanto, el uso de la HCR-20 es pertinente para la Psicología y la Psiquiatría Forenses, la Medicina Legal en general y las disciplinas relacionadas. El instrumento tiene la estructura de una escala que permite cotejar los factores de riesgo del comportamiento violento. Contiene 20 ítems organizados de la siguiente manera: 10 factores pasados (Factores Históricos), 5 variables actuales o presentes (Factores Clínicos) y 5 aspectos futuros (Factores de Riesgo Medioambientales). Suministra definiciones operativas y criterios de valoración. A los efectos de investigación se utiliza como escala intervalar cuya puntuación total mínima es 0 y la máxima 40. Estudios en otros países dan cuenta de su confiabilidad y validez (Belfrage, 1998; Belfrage et al., 2004; de Borba Telles et al., 2009a; Douglas et al., 1996; Douglas et al., 2003; Folino et al., 2004c; Folino et al., 2005; Folino et al., 2007b; Folino, 2015; Penney et al., 2014).

La SARA es una guía clínica diseñada originalmente en Canadá para cotejar los factores de riesgo de violencia conyugal (Kropp et al., 1995). En este estudio se utilizó la versión traducida y adaptada en Argentina (Folino, 2004a). Está constituida por 20 ítems agrupados en las siguientes secciones: Antecedentes penales; Adaptación psicosocial; Historia de violencia conyugal; Último delito; Otros. Cada ítem debe ser evaluado en su presencia, ya sea parcial o ausencia y, también, en cuando a si configura un ítem decisivo en la conclusión respecto al riesgo inminente de violencia. El instrumento propone la comunicación de las conclusiones en términos de bajo, moderado o alto riesgo inminente de daño al cónyuge y a terceros identificables (hijos, otros familiares, nueva pareja de un cónyuge anterior) en base a un proceso de juicio profesional. Estudios previos soportan su confiabilidad y validez (Andrés Pueyo et al., 2002; Kropp \& Hart, 2000; Messing \& Thaller, 2013).

El Hare Psychopaty Checklist - Revised -PCL-R- es un instrumento para evaluar características psicopáticas de la personalidad (Hare, 2003a). Consta de 20 ítems, con alternativas de puntuación 0.1 y 2 ; tiene un rango de puntuación total entre 0 y 40 y la posibilidad de prorrateo para cuando se omiten algunos ítems; el puntaje de corte para el diagnóstico de psicopatía según el manual es de 30 puntos y el mismo se sostiene en algunas investigaciones latinoamericanas ( Folino \& Hare, 2005; León-Mayer et al., 2014a; León-Mayer et al., 2015). En el manual se describen dos factores, uno que representa las características afectivas e interpersonales y otro que captura los aspectos conductuales de la psicopatía. Estos dos factores se subdividen en cuatro facetas definidas como: Interpersonal, que capta de manera más específica la forma en que el sujeto se relaciona con otras personas; Afectiva, que evalúa la resonancia afectiva de la persona frente a los diferentes acontecimientos en su vida y la calidad de su vinculación con los demás; Estilo de vida, que concentra características con el patrón de conducta impulsivo, irresponsable y parasitario; Antisocial, que es el que capta los problemas conductuales infanto-juveniles y la versatilidad criminal propia de la psicopatía (Hare, 2003a; León-Mayer et al., 2016). En este estudio se utilizó la versión chilena autorizada para investigación que cuenta con evidencias de su confiabilidad y validez (León-Mayer et al., 2010, 2013, 2014b, 2015). El instrumento cuenta con el sostén empírico de estudios de América Latina que informan valores del coeficiente de correlación intraclase entre 0.90 y 0.99 para las puntuaciones totales y de los factores; valores de kappa entre 0.78 y 0.90 para el diagnóstico categórico y valores del coeficiente de Cronbach entre 0.83 y 0.99 (Folino et al., 2003; Folino \& Hare, 2005; Folino \& Castillo, 2006). También hay diversos estudios que sostienen la confiabilidad de versiones en otros idiomas (Grann et al., 1998; Laurell \& Daderman, 2007; Pham, 1998; Sullivan et al., 2006).

Estadísticas: Los análisis estadísticos se realizaron con SPSS (IBM Corp. SPSS Inc., 2011). Se estimaron los coeficientes de correlación intraclase con los puntajes independientes del correspondiente par de evaluadores y se calcularon estadísticos descriptivos y de congruencia interna con los puntajes consensuados. 
Reparos éticos: El proyecto de estudio cuenta con la aprobación de las diferentes instancias de la Facultad de Jurisprudencia y Ciencias Políticas y Sociales de la Universidad de Cuenca y de la Secretaría de Educación Superior, Ciencia, Tecnología e Innovación del Ecuador. La obtención de información se realizó con el consentimiento de los entrevistados durante las actividades rutinarias del Instituto.

\section{RESULTADOS}

Fueron evaluados 20 (54\%) mujeres y 17 (46\%) varones cuyos promedios de edad fueron 32 y 33 años respectivamente. Una de las personas tenía nacionalidad brasilera, las restantes ecuatoriana. El promedio de años de escolaridad fue 12.5 (DE 7.1) años.

Hubo un único caso derivado de institución Penal; el resto fue derivado por instituciones judiciales en lo civil. Las conductas motivantes de la intervención judicial se relacionaron con violencia familiar predominantemente, si bien cabe destacar que hubo dos casos en los que la conducta fue un delito contra la integridad sexual y dos casos de abandono de persona. En cada caso, la fuente de derivación solicitó uno, dos o tres tipos de servicios al instituto. El primer servicio requerido se distribuyó de la siguiente manera: Terapia de familia (40.5\%); Terapia individual (21.6\%); Orientación familiar (18.9\%); Terapia de pareja (10.8\%) y Evaluación psicológica (8.1\%). De los tres casos en los que se requirió como primer servicio Evaluación psicológica, dos tuvieron el adicional pedido de alguna forma de intervención, por lo que, se verificó que sólo en un caso no fue requerida alguna forma de intervención del plantel profesional. Este resultado pone de manifiesto el rol de prestador de servicios de intervención psicológica y social que tiene el instituto, lo que implica un plus relevante a la prestación de servicios de evaluación que tradicionalmente se realiza en los sistemas forenses.

Los casos fueron valorados con los instrumentos independientemente y luego de manera consensuada. Respecto a los coeficientes de correlación intraclase calculados con las puntuaciones independientes, las categorías siguientes son las que suelen utilizarse para evaluar la confiabilidad observada (Fleiss, 1986): valor mayor o igual que $0.75=$ excelente; valor entre 0.40 y $0.74=$ aceptable a buena; valor menor a $0.40=$ pobre. Los resultados expuestos en la Tabla 1 , indican que la concordancia entre evaluadores para diversas sub-escalas se ubica en la categoría de excelente. La concordancia para la faceta antisocial del PCL-R y para la sub escala de R de la HCR-20 se ubicó en la categoría de buena.

En los resultados consensuados con PCL-R, HCR 20 y SARA (Tabla 1) se destaca la verificación de valores promedios muy por debajo de los que suelen obtenerse en población penitenciaria. Este perfil, que será discutido en la sección siguiente, ofrece información valiosa a la hora de planificar las intervenciones. Por otra parte, dadas las características de escala aditiva del PCL-R, también fueron evaluados otros aspectos de la confiabilidad: el coeficiente de Cronbach para el total de la escala fue 90. Habiéndose omitido el ítem 19 relacionado con el fracaso en previa libertad condicional o medida de supervisión judicial por tener numerosas omisiones, se calculó la correlación ítem-total. Todos los ítems tuvieron correlación mayor que 0.35 , indicando que contribuyen significativamente con la puntuación total del PCL-R; la media de correlaciones ítem-total fue 0.62.

La valoración de riesgo se realizó siguiendo el procedimiento de la guía SARA que se adicionó a la documentación exhaustiva generada con los restantes instrumentos y variables ad hoc. La Figura 1 representan los resultados de valoración de riesgo de violencia consensuado teniendo en cuenta dos tipos de potenciales blancos: la violencia hacia la pareja y la violencia hacia terceros. La distribución de proporciones pone de manifiesto que la población estudiada tiene predominio de bajo a moderado riesgo para ambos potenciales blancos y que la mayor proporción con riesgo de alto nivel de violencia se da en el contexto conyugal.

La concordancia entre las evaluaciones individuales estimadas con el CCI fue excelente para la evaluación de riesgo de violencia hacia la pareja: 0.76 para las psicólogas y 0.90 para las trabajadoras sociales. La concordancia de la evaluación de riesgo hacia terceros se ubicó en la categoría de buena: 0.63 para psicólogas y 0.62 para trabajadoras sociales. 
Tabla 1. Puntuaciones consensuadas y concordancia entre puntuaciones independientes con PCL-R, HCR-20 y SARA.

\begin{tabular}{lcccccc}
\hline Factor & Mínimo & Máximo & Media & DE & CCI $^{\mathrm{a}}$ & CCI $^{\mathrm{b}}$ \\
\hline PCL-R Interpersonal & 0 & 7 & 2.1 & 2.1 & 0.82 & - \\
PCL-R Afectivo & 0 & 8 & 2.7 & 2.9 & 0.93 & - \\
PCL-R Conductual & 0 & 12 & 3.2 & 2.6 & 0.84 & - \\
PCL-R Antisocial & 0 & 8 & 2.5 & 2 & 0.49 & - \\
PCL-R Total & 1 & 30 & 10.4 & 8 & 0.96 & - \\
H Total & 0 & 14 & 4.3 & 3.5 & 0.86 & - \\
C Total & 0 & 8 & 3.2 & 2.1 & 0.90 & - \\
R Total & 0 & 8 & 4.6 & 2.1 & - & 0.75 \\
HCR-20 Total & 0 & 28 & 12.1 & 6.2 & - & - \\
SARA Antecedentes penales & 0 & 6 & 1.3 & 1.6 & 0.85 & 0.90 \\
SARA Adaptación psicosocial & 0 & 10 & 3.8 & 2.7 & 0.89 & $0.83^{\mathrm{c}}$ \\
SARA Historia de violencia & 0 & 11 & 4.5 & 3.1 & 0.77 & 0.90 \\
conyugal & & & & & & \\
SARA Último delito & 0 & 4 & .9 & 1.3 & 0.86 & 0.94
\end{tabular}

Nota. N: 37. DE: Desvío estándar. CCI: Coeficiente de Correlación Intraclase, medida singular calculada para el modelo de efectos mixtos y con las puntuaciones independientes. Las estadísticas descriptivas se basan en las puntuaciones consensuadas. a: corresponde a dupla de psicólogas. b: corresponde a dupla de trabajadoras sociales. c: cálculo en base a suma de ítems 4 y 5.

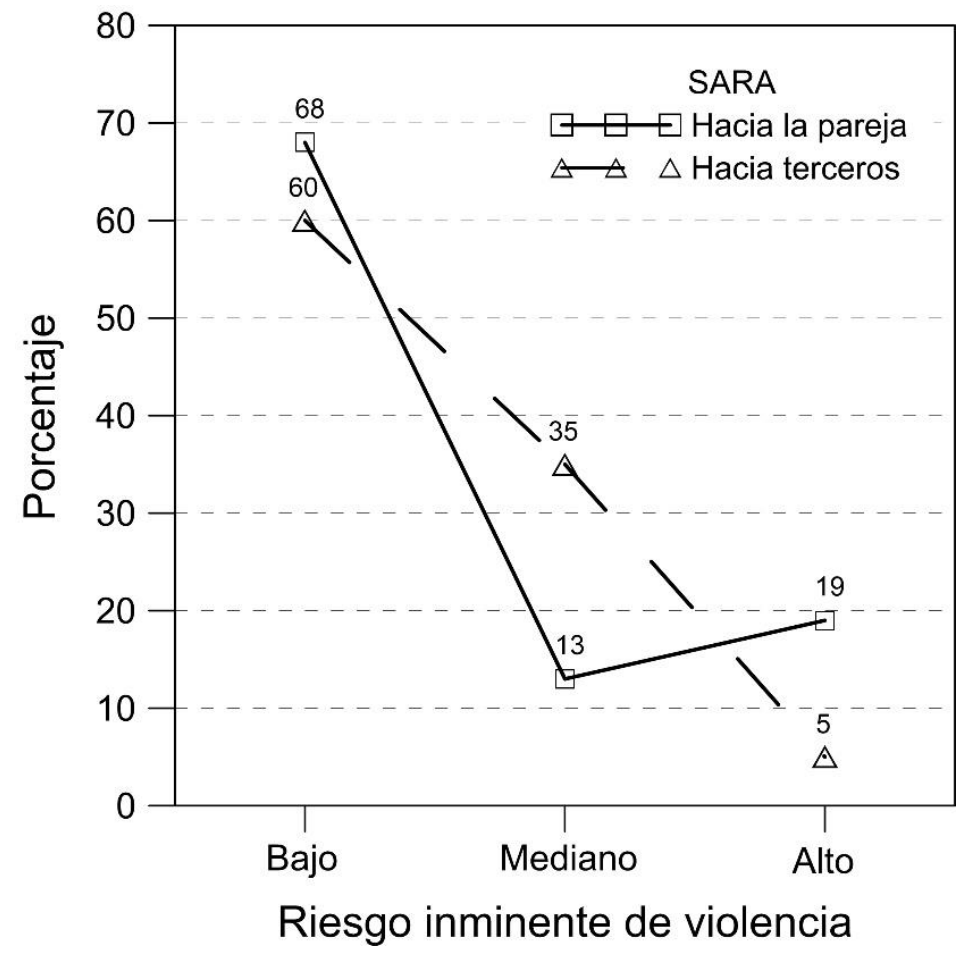

Figura 1. Conclusión consensuada sobre el riesgo inminente de violencia hacia la pareja y terceros utilizando SARA. 


\section{DISCUSIÓN}

La meta planteada en este estudio fue contribuir a sistematizar la evaluación de riesgo de violencia en el Ecuador poniendo a prueba la confiabilidad de instrumentos de evaluación de psicopatía e instrumentos de juicio profesional estructurado para la evaluación de riesgo de violencia en una institución universitaria que brinda servicios forenses en Cuenca. Para una completa discusión de los resultados será conveniente, en principio, considerar la significación que el procedimiento tuvo en el contexto de ejecución. Al comparar con prácticas precedentes (Ullauri et al., 2016), surge un contraste que pone en evidencia la factibilidad de sistematizar la tarea de evaluación en el servicio y la incorporación de estrategias tendientes a optimizar la transparencia de procedimientos y actualizar las tácticas empleadas. Este tipo de sistematización ofrece vías de desarrollo importantes ya que facilita la mensura de los hallazgos y las comparaciones entre ámbitos y períodos distintos, además de contribuir a la solvencia en las fundamentaciones. Por lo tanto, cabe destacar que el proyecto cumplió con esta parte de su meta.

En relación al objetivo específico de poner a prueba la confiabilidad de los instrumentos, se puede afirmar que los resultados obtenidos permiten sostener la confiabilidad de su uso en el ámbito forense ecuatoriano. Los resultados para el PCL-R, con la excepción la faceta Antisocial, fueron categorizados como excelentes, como ocurrió con los obtenidos en Argentina (Folino et al., 2003; Folino \& Castillo, 2006) y en Chile (León-Mayer et al., 2014a). La concordancia entre evaluadores respecto a la faceta Antisocial no alcanzó el nivel de excelente como hubiera sido deseable, sino el nivel de bueno. Si bien no genera desmedro en la apreciación general de la confiabilidad del instrumento, el hallazgo amerita extender la discusión. Que la población analizada no tenga elementos tan notorios del componente antisocial como los tiene, por ejemplo, una población penitenciaria, posiblemente repercuta en que la captación por el clínico sea una tarea más compleja y, por lo tanto, más expuesta a dispersión de las valoraciones. Independientemente de ello, el hallazgo abre la puerta a futuros estudios.

Respecto al HCR-20 y al SARA, es necesario tener presente que, en su aplicación a nivel individual, los factores de riesgo evaluados deben ser considerados a la luz del juicio profesional y no de manera cuantitativa o directamente aditiva. Su consideración desde una perspectiva cuantitativa, como variable de nivel intervalar, resulta valiosa para investigaciones a nivel poblacional más no a casos individuales. Por ejemplo, un uso correcto de estos métodos permitiría detectar el tipo de factores predominantes a los efectos de la planificación institucional de intervención, además de explorar su concordancia, como se hace en este estudio.

Habiendo expresado el reparo metodológico, la interpretación de los resultados orienta también a sostener la confiabilidad de los instrumentos. Coincidentemente con estudios previos (Folino et al., 2004c), los valores de la concordancia para el HCR-20 se ubicaron en la categoría de excelentes. Los valores obtenidos para el SARA en las subsecciones también se ubicaron en la categoría de excelente e, incluso, son más altos que los informados en estudios previos (Kropp \& Hart, 2000). La concordancia en la conclusión sobre el riesgo de violencia hacia la pareja fue también excelente. Aunque sin alcanzar a afectar la interpretación de confiabilidad, la concordancia con el riesgo de violencia hacia terceros resultó solamente buena. Lamentablemente no se cuenta con otros estudios latinoamericanos que informen sobre este detalle; si bien es probable que la especificidad de los factores, o las características de la muestra, hayan influido en una mayor concordancia respecto al riesgo de violencia conyugal, este aspecto queda como tema para revisión en estudios futuros. La interpretación de los hallazgos sobre la confiabilidad en este estudio y la consideración conjunta de evidencias de la validez del SARA proveniente de otras investigaciones (Belfrage et al., 2012; Messing \& Thaller, 2013) constituyen fuertes argumentos para su utilización sistemática en las evaluaciones forenses e intervenciones sobre la violencia familiar.

Allende los resultados que sostienen la confiabilidad de los métodos utilizados, es menester resaltar que, tal como lo expresan los respectivos manuales, para una precisa aplicación resulta imprescindible el correspondiente entrenamiento y el cumplimiento del procedimiento por parte de personal con apropiadas acreditaciones. En ciencias de la conducta humana difícilmente se encuentra un instrumento que en su aplicación sea invulnerable a la negligencia. Para una precisa evaluación deben confluir la táctica confiable, la capacitación para llevarla a cabo y la información necesaria. 
Los hallazgos de este estudio en el procedimiento de puntuaciones consensuadas también conforman una información valiosa relacionada con la validez del método de evaluación de psicopatía. $\mathrm{Si}$ bien deben reconocerse reparos en estas consideraciones, pues el estudio no se propuso revisar la validez discriminante, cabe destacar que las puntuaciones promedio obtenidas se encuentran dentro de lo sería esperable para el tipo de la población estudiada: bien por debajo de los promedios de población de penados y más cerca de la población con problemas de salud mental forense. Como ejemplos baste mencionar que mientras en el presente estudio el promedio PCL-R total fue de 10.4, en población penitenciaria chilena fue 20.9 (León-Mayer et al., 2014b), en población penitenciaria argentina fue 20.2 (Folino \& Hare, 2005) y en población forense brasilera fue 13.5 (de Borba Telles et al., 2012). Lamentablemente no se cuenta con información latinoamericana respecto a la distribución de los puntajes en comunidad, pero la revisión de Hare en páginas 52-53 incluye estudios de otros países que encontraron un promedio de 8.8 en grupos control comunitarios (Hare, 2003b).

Por otra parte, dada la condición de escala aditiva del PCL-R, que permite cuantificación en la aplicación personal y utilización de puntaje de corte, los valores consensuados también permitieron estimar otros indicadores de confiabilidad, como el coeficiente de Cronbach y la correlación ítem-total. Los hallazgos permiten sostener la congruencia interna del instrumento y son consistentes con previos estudios latinoamericanos (Folino \& Castillo, 2006; León-Mayer et al., 2010; León-Mayer et al., 2014b) y con los que forman la base agregada informada en la segunda versión del manual (Hare, 2003b).

El presente estudio tiene diversas implicaciones en la práctica profesional ecuatoriana. A partir de la disponibilidad de instrumentos que tienen soporte empírico respecto a su confiabilidad, las evaluaciones forenses podrían realizarse de manera más sistemática. Los constructos y procedimientos definidos operacionalmente en instrumentos que son utilizados actualmente en múltiples partes del mundo permitirían mayor precisión a la hora de verificar los factores de riesgo en los casos, comunicarlos a los interesados, autoridades judiciales o colegas involucrados en la intervención. Todo ello redundaría en un importante resguardo a la responsabilidad profesional en tanto disminuye el riesgo de evaluaciones idiosincráticas y de omitir información basada en evidencias.

Asimismo, en tanto diversos factores de riesgo son dinámicos, es decir, varían a lo largo del tiempo agravándose o atenuándose, y son pausibles de tratamientos, la disponibilidad de las medidas sistemáticas, cuya confiabilidad sostiene el presente estudio, será valiosa para revisar el impacto de algún programa de intervención o la variación debida a determinado cambio legislativo o institucional. En síntesis, que la sistemática implementada resulte confiable transciende el desafío del proyecto. Esa información se constituye en un capital para la práctica profesional, en un área socialmente sensible como es la violencia conyugal, y para nuevas investigaciones.

El estudio tiene fortalezas y debilidades. Entre las primeras, se destaca la amplia experiencia en evaluación y tratamiento a población local con que cuentan los co-autores ecuatorianos. La población local está conformada por grupos étnicos diversos que presentan especiales modismos comunicacionales, costumbre y valores, de tal manera que resulta imprescindible contar con profusa experiencia local para tener precisión en la interpretación de la realidad social. Otra fortaleza recae en el diseño y en los instrumentos que ya cuentan con respaldo empírico de otras regiones. Entre las debilidades se cuenta la de no haber utilizado medidas de auto-informadas que podrían haber permitido explorar la validez concurrente a partir de un distinto modo de obtención de información.

Finalmente, es particularmente destacable que los resultados transmitidos surgen de un estudio realizado en una institución universitaria solventada por una agencia nacional de investigación. El detalle no es menor, estudios internacionales recientes señalan la necesidad de achicar la brecha entre el conocimiento actual sobre la violencia y las prácticas de evaluación e intervención (Arbach-Lucioni et al., 2015; Cote et al., 2012; Folino et al., 2016; Goldstein et al., 2015; Singh et al., 2013). El achicamiento de esa brecha, el desarrollo de nuevo conocimiento tendiente a disminuir la violencia y a mitigar sus consecuencias, es difícilmente alcanzable sin el interés del estado y de la academia. Los autores aspiran a que éste modesto aporte resulte un ejemplo de lo que puede lograrse cuando confluyen sectores oficiales y académicos en la lucha contra la violencia. 


\section{CONCLUSIONES}

La investigación permitió obtener diversas conclusiones trascendentes para América Latina: La confiabilidad de las versiones en español del PCL-R, el SARA y el HCR-20 fue puesta a prueba en Ecuador y sostenida. La sistematización del proceso de evaluación de riesgo de violencia pudo ser lograda a través del adecuado entrenamiento y del cuidadoso uso de esos instrumentos. Este estudio deja establecida una motivante heurística e impulsa, en la interfaz del Derecho y la Salud Mental, un estilo de práctica profesional con base empírica y transparencia metodológica. Seguramente, todo ello promoverá resguardo de la responsabilidad profesional y mejor provisión de servicio.

\section{BIBLIOGRAFÍA}

Acero González AR, Escobar Córdoba F, Castellano Castañeda G (2007). Factores de riesgo para violencia y homicidio juvenil. Revista Colombiana de Psiquiatría 36:78-97

Alarcón P, Wenger L, Chesta S, Salvo S (2012). Validez predictiva del instrumento Evaluación de Riesgos y Recursos ( FER-R ) para la intervención en adolescentes infractores de ley: estudio preliminar. Universitas Psychologica 11(4):1183-1195

Andrés Pueyo A, López S, Alvarez E (2008). Valoración del riesgo de violencia contra la pareja por medio de la SARA. Papeles del Psicólogo 29(1):107-122

Arbach-Lucioni K, Desmarais S, Hurducas C, Condemarin C, Dean K, Doyle M, Folino J, Gody Cervera V, Grann M, Ho MYR, Large MM, Pham TH, Hjort Nielsen L, Rebocho MF, Reeves KA, Rettenberger M, de Ruiter C, Seewald K, Singh JP (2015). La práctica de la evaluación del riesgo de violencia en España. Revista de la Facultad de Medicina 63(3):357-366

Asamblea Constituyente (2008). Constitución de la República del Ecuador. Quito, Ecuador: Registro Oficial $\mathrm{N}^{\circ} 449$

Asamblea Nacional República del Ecuador (2014). Código Orgánico Integral Penal. Quito, Ecuador: Registro Oficial $\mathrm{N}^{\circ} 180$

Belfrage H (1998). Implementing the HCR-20 scheme for risk assessment in a forensic psychiatric hospital: Integrating research and clinical practice. Journal Forensic Psychiatry 9(2):328-338

Belfrage H, Fransson G, Strand S (2000). Prediction of violence using the HCR-20: A prospective study in two maximum-security correctional institutions. Journal Forensic Psychiatry 11(1):167-175

Belfrage H, Strand S, Storey JE, Gibas AL, Kropp PR, Hart SD (2012). Assessment and management of risk for intimate partner violence by police officers using the Spousal Assault Risk Assessment Guide. Law and Human Behavior 36(1):60-67. https://doi.org/10.1037/h0093948

Claix A, Pham TH (2004). Evaluation of the HCR-20 violence risk assessment scheme in a Belgian forensic population. Encephale 30(5):447-453

Congreso Nacional (2003). Código de la niñez y adolescencia. Quito, Ecuador: Registro Oficial 737 del 3 de Enero de 2003

Consejo Universitario de la Universidad de Cuenca (1984). Oficio Nro 84 del Rectorado, Resolución del Consejo Universitario de la Universidad de Cuenca, Cuenca

Consejo Universitario de la Universidad de Cuenca (2011). Resolución del Consejo Universitario de la Universidad de Cuenca del 18 de Octubre de 2011, Cuenca

Cote G, Crocker AG, Nicholls TL, Seto MC (2012). Risk assessment instruments in clinical practice. Canadian Journal of Psychiatry 57(4):238-244

de Borba Telles LE, Day VP, Folino JO, Taborda JGV (2009a). Reliability of the Brazilian version of HCR-20 assessing risk for violence. Revista Brasileira de Psiquiatria 31:253-256

de Borba Telles LE, Folino JO, Taborda JGV (2009b). Incidência de conduta violenta e antissocial em 
população psiquiátrica forense. Revista fr Psiquiatria de Rio Grande do Sul 33:3-7

de Borba Telles LE, Folino JO, Taborda JGV (2012). Accuracy of the historical, clinical and risk management scales (HCR-20) in predicting violence and other offenses in forensic psychiatric patients in Brazil. International Journal of Law and Psychiatry 35(5-6):427-431

de Borba Telles LE, Taborda JGV, Folino JO (2010). Avanços na avaliação de risco de violência [Progress on violence risk assessment]. Multijuris 5:36-43

de Vogel V, de Ruiter C, Bouman Y, de Vries Robbe M (2007). Guide to the SAPROF. Structured assessment of protective factors for violence risk. Version 1. Utrecht: Forum Educatief

Douglas KS, Hart SD, Webster CD, Belfrage H (2013). HCR-20 v3 Assessing risk for violence. Vancouver: Mental Health, Law, and Policy Institute, Simon Fraser University

Douglas KS, Hart SD, Webster CD, Belfrage H (2015). HCR-20 v3: Valoración del riesgo de violencia: guía del evaluador. (Arbach-Lucioni K, Andres-Pueyo A, Eds.) (Adaptación). Barcelona: Universitat de Barcelona

Douglas KS, Kropp PR (2002). A Prevention-based paradigm for violence risk assessment - Clinical and research applications. Criminal Justice and Behavior 29(5):617-658

Douglas KS, Ogloff JRP (2003). Multiple facets of risk for violence: The impact of judgmental specificity on structured decisions about violence risk. International Journal of Forensic Mental Health 2(1):19-34

Douglas KS, Ogloff JRP, Hart SD (2003). Evaluation of a model of violence risk assessment among forensic psychiatric patients. Psychiatric Services (Washington DC) 54(10):1372-1379

Douglas KS, Webster CD, Wintrup A (1996). The HCR:20 risk assessment scheme: Psychometric properties in two samples. Paper presented at the Annual Conference of the American Psychological Association, Toronto

Ennis BJ, Litwack TR (1974). Psychiatry and the presumption of expertise: Flipping coins in the courtroom. California Law Review 62:693-752

Escobar-Córdoba F, Acero-González AR, Folino JO (2015). Homicidas juveniles en Bogotá, estudio de grupos focales. Revista de la Facultad de Medicina 63(3):389-398

Falzer PR (2013). Valuing structured professional judgment: predictive validity, decision-making, and the clinical-actuarial conflict. Behavioral Sciences \& the Law 31(1):40-54

Fleiss JL (1986). The design and analysis of clinical experiments. New York: Wiley

Folino JO (2003). Evaluación de riesgo de violencia -HCR - 20-Versión en español, adaptada y comentada. La Plata, Argentina: Interfase Forense

Folino JO (2004a). Guía para la evaluación del riesgo de violencia conyugal -SARA-Versión en español adaptada y comentada. La Plata: Editorial Interfase Forense

Folino JO (2004b). Nuevos paradigmas en la evaluación de peligrosidad [New paradigms in dangerousness assessment]. Doctrina Judicial - Editorial La Ley XX:871-876

Folino JO (2005). Risk assessment and violent recidivism risk management in convicts from Argentina. Research in Social Problems and Public Policy 12:75-78

Folino JO (2015). Predictive efficacy of violence risk assessment instruments in Latin-America. European Journal of Psychology Applied to Legal Context 7(2):51-58

Folino JO, Marchiano S (2002). Tasa basal de recidiva delictiva. Intercambios (5). Retrieved from http://der.jursoc.unlp.edu.ar/intercambios/index.htm

Folino JO, Arado M, Ferrari L, Marengo M (2002). Manejo del riesgo de recidiva violenta y el abuso de sustancias. La Plata: Procuración General de la Suprema Corte de Justicia, Provincia de Buenos Aires

Folino JO, Astorga C, Sifuentes M, Ranze S, Tenaglia D (2003). Confiabilidad de la Hare Psychopathy Checklist-Revised en población psiquiátrico forense argentina. Revista Argentina de Clínica Neuropsiquiátrica 10(5):5-11

Folino JO, Escobar-Córdoba F (2004). Nuevos aportes a la evaluación de riesgo de violencia. 
MedUNAb -Especial Salud Mental - Revista de la Facultad de Medicina de la UNAB 7:99-105

Folino JO, Arado MG, Ferrari LA, Marengo M (2004a). Prevención de recidiva delictual en abusadores de sustancias. Revista Médica de La Plata 38(3):20-24

Folino JO, Marengo CM, Marchiano SE, Ascazibar M (2004b). The Risk Assessment Program and the Court of Penal Execution in the Province of Buenos Aires, Argentina. International Journal of Offender Therapy and Comparative Criminology 48:49-58

Folino JO, Castillo JL, Cáceres MS, Campos ML, Silveri M, UcínS (2004c). Confiabilidad de la versión argentina de la HCR-20. Medicina Forense Argentina, Boletín de La Asociación de Médicos Forenses de laa República Argentina 27:2-5

Folino JO, Hare RD (2005). Listado revisado para verificación de la psicopatía: su estandarización y validación en la Argentina. Acta Psiquiátrica y Psicológica de América Latina 51:94-104

Folino JO, Cáceres MS, Campos ML, Silveri M, Ucín S, Ascazibar M (2005). Evaluación de factores dinámicos de riesgo de violencia. Vertex 16:188-195

Folino JO, Castillo JL (2006). Las facetas de la psicopatía según la Hare Psychopathy Checklist Revised y su confiabilidad. Vertex 17, 325-330.

Folino JO, Almirón MP, Ricci MA (2007a). Factores de riesgo de recidiva violenta en mujeres filicidas. Vertex 18(74):258-267

Folino J, Almiron M, Ricci MA (2007b). Violent recidivism risk factor in filicidal women. Vertex $18(74): 258-267$

Folino JO, Villaba DK, Singh JP (2016). Violence risk assessment in Latin America. In: Singh JP, Bjørkly S, Fazel S (Eds.), International perspectives on violence risk assessment (pp. 181-191). New York: Oxford University Press

Goldstein NES, Houck MJ, Folino JO (2015). Tópicos actuales de la investigación internacional sobre riesgo de violencia. Criminalidad 57(1):27-43

Grann M, Langstrom N, Tengstrom A, Stalenheim EG (1998). Reliability of file-based retrospective ratings of psychopathy with the PCL-R. Journal of Personality Assessment 70(3):416-426

Gutierrez A, Wiese R, Castillo J, Folino JO (2012). Evaluación de características psicométricas de la versión argentina de la Hare Psychopathy Checklist: Youth Version. Vertex 23(103):180-187

Hanson KR (1997). The development of a brief actuarial risk scale for sexual offense recidivism. Ottawa, Ontario, Canada: Public Works and Government Services Canada

Hare RD (2003a). The Hare Psychopathy Checklist - Revised ( $2^{\text {nd }}$ ed.). Technical Manual. Toronto: Multi-Health Systems Inc

Hare RD (2003b). The Hare Psychopathy Checklist - Revised Manual (2 $2^{\text {nd }}$ ed.). Toronto: Multi-Health Systems Inc

Hare RD, Hart SD (1993). Psychopathy, mental disorder and crime. In: Hodgins S (Ed.), Mental disorder and crime (pp. 104-115). London: Sage

Harris GT, Rice ME, Cormier CA (1991). Psychopathy and violent recidivism. Law and Human Behavior 15:625-637

Harris GT, Rice ME, Quinsey VL (1993). Violent recidivism of mentally disordered offenders: The development of a statistical prediction instrument. Criminal Justice and Behavior 20(4):315-335

Harris GT, Rice ME, Quinsey VL, Cormier CA (2015). Violent offenders: Appraising and managing risk ( $3^{\text {rd }}$ ed.). Washington DC: American Psychological Association

IBM Corp. SPSS Inc. (2011). IMB SPSS Sstatistics (20). United States: SPSS Inc

Kropp PR, Hart SD (2000). The spousal assault risk assessment (SARA) guide: Reliability and validity in adult male offenders. Law and Human Behavior 24(1):101-118

Kropp PR, Hart SD, Webster CD, Eaves D (1995). Manual for the spousal assault risk assessment guide $\left(2^{\text {nd }} e d.\right)$. Vancouver: British Columbia Institute on Family Violence

Laurell J, Daderman AM (2007). Psychopathy (PCL-R) in a forensic psychiatric sample of homicide offenders: some reliability issues. International Journal of Law and Psychiatry 30(2):127-135 
León-Mayer E (2009). Hare PCL-R versión chilena autorizada para investigación. Viña del Mar, Chile

León-Mayer E, Asún Salazar D, Folino JO (2010). Confiabilidad y validez de la versión chilena de la Hare PCL-R. Revista de la Facultad de Medicina de la UNAL 58:103-114

León-Mayer E, Cortés Olavarría MS, Folino JO (2014a). Descripción multidimensional de población carcelaria chilena. Psicoperspectivas 13:68-81

León-Mayer E, Folino J, Hare R (2014b). Confiabilidad de la versión chilena de la Hare PCL-R y validez convergente con otros instrumentos de evaluación de psicopatía. Vertex 25(116):245-252

León-Mayer E, Folino JO, Neuman C, Hare R (2015). The construct of psychopathy in Chilean prison population. Revista Brasileira de Psiquiatria 37(3):191-196

León-Mayer E, Folino JO, Taborda JG, Hare RR (2016). Violencia e psicopatía. In: Abdala-Fillo E, Chalub M, de Borba Telles L (Eds.), Psiquiatria forense (Terceira, pp. 529-540). Porto Alegre: Grupo Artmed Editora Ltda

León-Mayer E, Neuman C, Hare RR, Folino JO (2013). Aproximación diagnóstica de psicopatía mediante instrumento autoinformado. Revista Criminalidad 55:251-264

León Mayer E, Folino JO (2009). Determinación del nivel de fiabilidad y validez del PCL-R en población forense masculina Chilena. Departamento de Postgrado Escuela de Psicología. Universidad de Valparaíso, Valparaíso, Chile

Link BG, Stueve A, Monahan J, Steadman H (1994). Psychotic symptoms and the violent/illegal behavior of mental patients compared to community controls. In: Mohana J, Steadman HJ (Eds.), Violence and Mental Disorder: Developments in Risk Assessment (pp. 137-159). Chicago: The University of Chicago Press

Messing JT, Thaller J (2013). The average predictive validity of intimate partner violence risk assessment instruments. Journal of Interperssonal Violence 28(7):1537-1558

Monahan J (1981). Predicting violent behaviour: An assessment of the clinical techniques. Beverly Hills, CA: Sage

Monahan J, Steadman HJ, Appelbaum PS, Robbins PC, Mulvey EP, Silver E, Roth LH, Grisso T (2000). Developing a clinically useful actuarial tool for assessing violence risk. British Journal of Psychiatry 176(4):312-319

Mossman D (1994). Assessing predictions of violence: Being accurate about accuracy. Journal Consulting and Clinical Psychology 62(4):783-792

Mulvey EP, Shaw E, Lidz CW (1994). Editorial: Why use multiple sources in research on patient in the community? Criminal Behavior and Mental Health 4:253-258

Ochoa-Rosales M (2007). Factores de riesgo en la población carcelaria del Cantón Cuenca Ecuador [Risk factors of the prison population in Cuenca district, Ecuador]. [Ms Cs dissertation] National University of La Plata, La Plata, Argentina

Ochoa J, Guillén X, Ullauri D, Narvaez J, León-Mayer E, Folino JO (2016). Relevancia de la evaluación del riesgo de violencia y de la psicopatía en el ámbito forense. Juris [submited]

Penney SR, McMaster R, Wilkie T (2014). Multirater reliability of the historical, clinical, and risk management-20. Assessment 21(1):15-27

Pérez-Luco R, Lagos L, Báez C (2012). Reincidencia y desistimiento en adolescentes infractores : análisis de trayectorias delictivas a partir de autorreporte de delitos, consumo de sustancias y juicio profesional. Universitas Psychologica 11(4):1209-1225

Pham TH (1998). Psychometric assessment of the PCL-R on an belgian prison sample. Encephale 24(5):435-441

Quinsey VL, Harris GT, Rice ME, Cormier CA (1999). Violent offenders - Appraising and managing risk ( $3^{\text {rd }}$ ed.). Washington DC: American Psychological Association

Quinsey VL, Rice ME, Harris GT (1995). Actuarial prediction of sexual recidivism. Journal of Interpersonal Violence 10:85-105

Rice ME, Harris GT (1992). A comparison of criminal recidivism among schizophrenic and 
nonschizophrenic offenders. International Journal of Law and Psychiatry 15(4):397-408

Rice ME, Harris GT, Lang C (2013). Validation of and revision to the VRAG and SORAG: The violence risk appraisal guide - Revised (VRAG-R). Psychological Assessment 25(3):951-965

Singh JP, Condemarín C, Folino JO (2013). El uso de instrumentos de evaluación de riesgo de violencia en Argentina y Chile [The use of violence risk assessment instruments in Argentina and Chile]. Revista Criminalidad 55(3):279-290

Singh JP, Grann M, Fazel S (2011). A comparative study of violence risk assessment tools: a systematic review and metaregression analysis of 68 studies involving 25,980 participants. Clinical Psychology Review 31(3):499-513

Steadman HJ, Mulvey EP, Monahan J, Robbins PC, Appelbaum PS, Grisso T, Roth LH, Silver E (1998). Violence by people discharged from acute psychiatric inpatient facilities and by others in the same neighbourhood. Archives of General Psychiatry 55:393-401

Sullivan EA, Abramowitz CS, Lopez M, Kosson DS (2006). Reliability and construct validity of the psychopathy checklist - revised for Latino, European American, and African American male inmates. Psychological Assessment 18(4):382-392

Swanson JW, Monahan J, Steadman H (1994). Mental disorder, substance abuse and community violence: An epidemiological approach. In: Monahan J, Steadman H (Eds.). Violence and mental disorder - Developments in risk assessment (pp. 101-136). Chicago: University of Chicago Press

Tardiff K (1989). A model for the short-term prediction of violence potential. In: Tardiff K, Brizer DA, Crowner ML (Eds.), Current approaches to the prediction of violence (pp. 3-12). Washington DC: American Psychiatric Press, Inc

Teitelbaum $\mathrm{P}$ (2010). Tasa basal de recidiva delictiva de los pacientes liberados post medida de seguridad del Instituto Psiquiátrico Forense de Porto Alegre, Brasil, en un período de 10 años (1994-2004) [Crime recidivism basal rate in released patients from the Forensic Psych. [Ms Cs dissertation] National University of La Plata, La Plata, Argentina

Ullauri D, Ochoa J, Guillén X, León-Mayer E, Folino JO (2016). Servicios universitarios en la interfaz del derecho y la salud mental: el caso de la Universidad de Cuenca, Ecuador. Criminalidad 57(3):103-117

Vasconcellos M, León Mayer E (2006). Aplicación de la escala diagnóstica de psicopatía (PCL-R) de $R$. Hare a un grupo de penadas de la Provincia de Valparaíso. Tesis de maestría no publicada, Escuela de Psicología, Universidad del Mar, Viña del Mar, Chile

Webster CD, Douglas KS, Eaves D, Hart SD (1997a). HCR - 20: Assessing risk for violence - Version 2. Burnaby: Mental Health, Law, and Policy Institute, Simon Fraser University

Webster CD, Douglas KS, Eaves D, Hart SD, Jackson MA (1997b). Predicting violence in mentally and personality disordered individuals. New York: Guilford

Webster CD, Eaves D, Douglas KS, Wintrup A (1995). The HCR-20 scheme: The assessment of dangerousness and risk. Burnaby, British Columbia: Simon Fraser University and Forensic Psychiatric Services Commission

World Health Organization (2014). Global status report on violence prevention 2014. Geneva: World Health Organization

Zúñiga DP, Mayer EL (2008). Caracterizació psicométrica del instrumento psychopathy checklist: Youth version (PCL:YV). Facultad de Educación y Humanidades Departamento de Psicología, Universidad de La Frontera, Temuco, Chile 